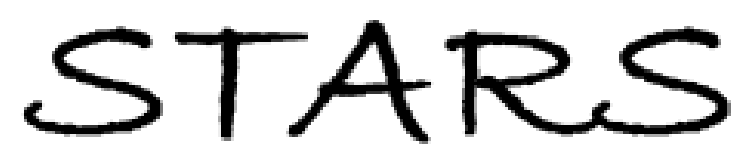

University of Central Florida

STARS

$1-1-2011$

\title{
Correlated electrical breakdown in arrays of high density aligned carbon nanotubes
}

\author{
Shashank Shekhar \\ University of Central Florida \\ Mikhail Erementchouck \\ University of Central Florida \\ Michael N. Leuenberger \\ University of Central Florida \\ Saiful I. Khondaker \\ University of Central Florida
}

Find similar works at: https://stars.library.ucf.edu/facultybib2010

University of Central Florida Libraries http://library.ucf.edu

This Article is brought to you for free and open access by the Faculty Bibliography at STARS. It has been accepted for inclusion in Faculty Bibliography 2010 s by an authorized administrator of STARS. For more information, please contactSTARS@ucf.edu.

\section{Recommended Citation}

Shekhar, Shashank; Erementchouck, Mikhail; Leuenberger, Michael N.; and Khondaker, Saiful I., "Correlated electrical breakdown in arrays of high density aligned carbon nanotubes" (2011). Faculty Bibliography 2010s. 1897.

https://stars.library.ucf.edu/facultybib2010/1897

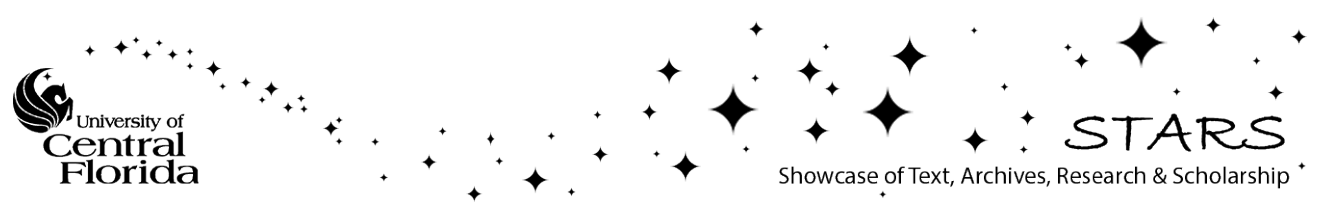




\section{Correlated electrical breakdown in arrays of high density aligned carbon nanotubes}

Cite as: Appl. Phys. Lett. 98, 243121 (2011); https://doi.org/10.1063/1.3600664

Submitted: 19 April 2011 . Accepted: 24 May 2011 . Published Online: 17 June 2011

Shashank Shekhar, Mikhail Erementchouk, Michael N. Leuenberger, and Saiful I. Khondaker

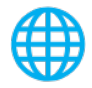

\section{ARTICLES YOU MAY BE INTERESTED IN}

High quality solution processed carbon nanotube transistors assembled by dielectrophoresis

Applied Physics Letters 96, 083110 (2010); https://doi.org/10.1063/1.3327521

Solution processed large area field effect transistors from dielectrophoreticly aligned arrays of carbon nanotubes

Applied Physics Letters 94, 113104 (2009); https://doi.org/10.1063/1.3100197

Electric-field-aligned growth of single-walled carbon nanotubes on surfaces

Applied Physics Letters 81, 3464 (2002); https://doi.org/10.1063/1.1518773

\section{Applied Physics Reviews} Now accepting original research 


\title{
Correlated electrical breakdown in arrays of high density aligned carbon nanotubes
}

\author{
Shashank Shekhar, Mikhail Erementchouk, Michael N. Leuenberger, and \\ Saiful I. Khondakerá) \\ Nanoscience Technology Center and Department of Physics, University of Central Florida, \\ 12424 Research Parkway, Orlando, Florida 32826, USA
}

(Received 19 April 2011; accepted 24 May 2011; published online 17 June 2011)

\begin{abstract}
We demonstrate that in a densely packed aligned array of single walled carbon nanotubes, the electrical breakdown of one nanotube leads to a highly correlated electrical breakdown of neighboring nanotubes, thereby producing a nanofissure. We show that the origin of the correlation is the electrostatic field of the broken nanotubes that produces locally inhomogeneous current and Joule heating distributions in the neighboring intact nanotubes triggering their breakdowns in the vicinity of the broken nanotubes. Our results suggest that the densely aligned arrays behave like a correlated solid. (C) 2011 American Institute of Physics. [doi:10.1063/1.3600664]
\end{abstract}

Due to their exceptional electronic properties including very high mobility, near ballistic conductance, and resistance against electromigration, single-walled carbon nanotubes (SWNTs) are considered to be a promising building block for future digital and analog electronics. ${ }^{1-3}$ Since it is difficult to control the chirality of individual nanotubes, arrays of SWNTs are becoming an important class of materials ${ }^{4-20}$ as they can (i) average out inhomogeneities of individual tubes, (ii) provide larger on currents, and (iii) reduce noise to provide higher cutoff frequency for radio frequency (rf) applications. Due to these advantages, there is a push to fabricate devices with massively parallel arrays of SWNTs for $\mathrm{rf}$ applications, ${ }^{4-6}$ field effect transistors, ${ }^{7-13}$ plastic electronics, ${ }^{7,8,14}$ display technologies, ${ }^{10,14}$ and sensors. ${ }^{14}$ However, since arrays contain a mixture of metallic and semiconducting nanotubes it is essential to selectively remove metallic nanotubes to obtain transistor properties. ${ }^{7-11,15}$ Such selective removal has been achieved for arrays containing up to $7 \mathrm{SWNT} / \mu \mathrm{m}$ assuming that the electrical breakdown of carbon nanotubes in air is due to Joule heating and oxidation. ${ }^{7}$ It was also assumed that individual nanotubes can be broken without affecting the neighboring nanotubes and that only metallic nanotubes can be selectively removed leaving semiconducting nanotubes in the network. Detailed investigation was not carried out to see how many nanotubes were left behind. In addition, the authors did not check if their assumptions remained valid for arrays of density higher than $7 \mathrm{SWNT} / \mu \mathrm{m}$. In other words, the aligned array was considered as a mere collection of individual nanotubes, which may not be the case when the nanotubes are in close proximity of each other.

A clear understanding of electrical breakdown of densely packed SWNT arrays is therefore essential for determining the fault-tolerance of nanotube arrays for electronic circuits. Since experimental and theoretical studies on individual SWNTs demonstrate that the breakdown is due to Joule heating and oxidation, which occurs at random places (at defect sites), a straightforward extrapolation of these studies to an array would suggest that the breakdown would occur at a

\footnotetext{
a) Author to whom correspondence should be addressed. Electronic mail: saiful@ucf.edu.
}

random point inside each nanotube. Here we show in our experiments on arrays of nanotubes that while this straightforward extrapolation is valid only for large internanotube separations (low density array), it does not hold for closely spaced nanotubes (high density array). We demonstrate that in a densely packed aligned array of SWNTs containing up to $30 \mathrm{SWNT} / \mu \mathrm{m}$, the electrical breakdown of one of the nanotubes leads to a highly correlated breakdown of neighboring nanotubes, thereby producing a nanofissure. Such a correlated electrical breakdown does not occur if the SWNTs are not densely packed in the array implying that the correlation depends strongly on the internanotube separation. We show theoretically that the correlated electrical breakdown is due to the electrostatic field of the broken nanotubes that produces locally inhomogeneous current and Joule heating distributions in the neighboring intact nanotubes triggering their breakdowns in the vicinity of the broken nanotubes.

To explore the correlated electrical breakdown, the SWNT aligned array containing $\sim 1$ to $\sim 30 \mathrm{SWNT} / \mu \mathrm{m}$ were assembled from high quality aqueous solution via dielectrophoresis between prefabricated $\mathrm{Pd}$ source and drain electrodes on $\mathrm{Si} / \mathrm{SiO}_{2}$ substrate. Figure 1(a) shows experimental setup for the SWNT assembly. Details of the

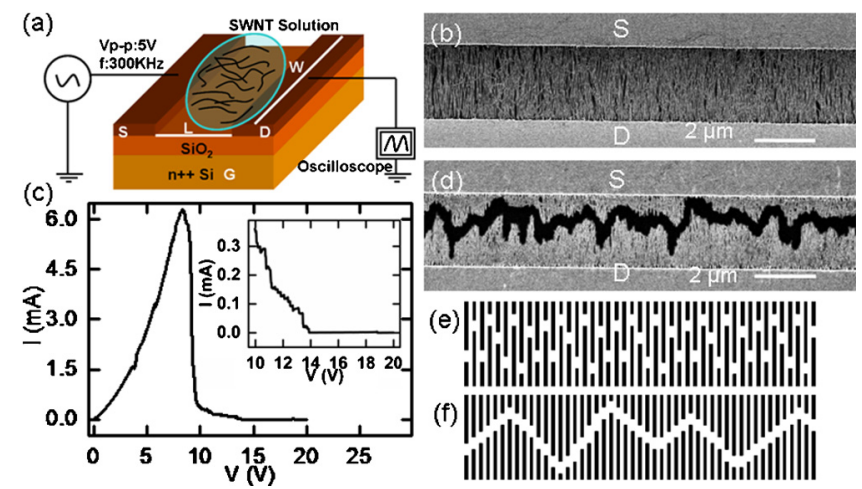

FIG. 1. (Color online) (a) Schematic of the SWNT assembly setup. (b) SEM image of an aligned array with $\sim 30 \mathrm{SWNT} / \mu \mathrm{m} . \mathrm{L}=2 \mu \mathrm{m}$ and $\mathrm{W}$ $=25 \mu \mathrm{m}$. (c) $I-V$ curve during electrical breakdown process with inset showing final stage of the breaking. (d) SEM image after breakdown shows nanofissure like continuous channel. The schematic of (e) random breakdown of nanotubes and (f) correlations in breakdown of adjacent nanotubes. 

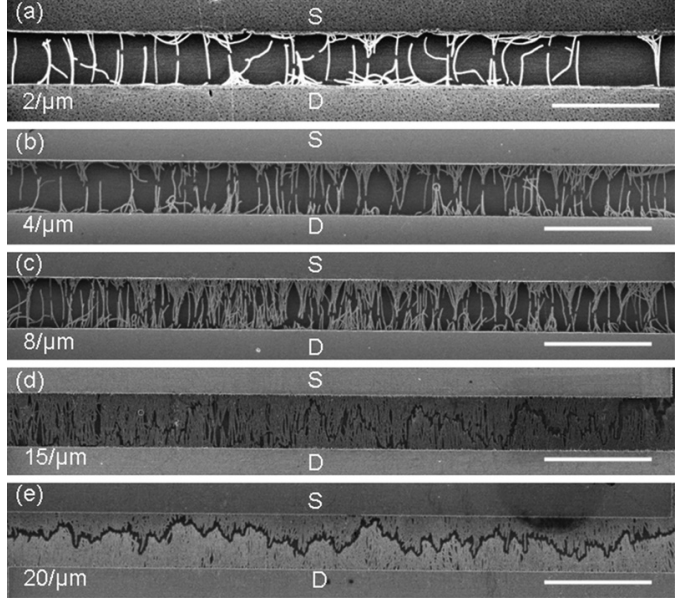

FIG. 2. Examination of correlated electrical breakdown of SWNTs by varying the linear density. (a) and (b) show the breaking of array with 2 and $4 \mathrm{SWNT} / \mu \mathrm{m}$, where breaking is random. (c) Breaking of $8 \mathrm{SWNT} / \mu \mathrm{m}$ and a pattern seems to appear. [(d) and (e)] The correlated patterns become more pronounced at densities 15 and $20 \mathrm{SWNT} / \mu \mathrm{m}$. The scale bars are $4 \mu \mathrm{m}$.

alignment can be found in Refs. 20 and 21. Figure 1(b) shows scanning electron micrographs of a densely packed SWNT aligned array with a linear density of $\sim 30 \mathrm{SWNT} / \mu \mathrm{m}$. The current $(I)$-voltage $(V)$ characteristics of this array [Fig. 1(c)] show that $I$ increases rapidly to a few milliamperes and then decreases to zero as the $V$ was increased. Scanning electron microscopy (SEM) images taken afterwards show interesting nanoscale fissure in the aligned nanotube network [Fig. 1(d)] occurred due to electrical breakdown of SWNTs.

The nanofissure shows that the breakdown of individual nanotubes in the array is not independent of each other. If they were independent, we would see random breakdown of individual nanotubes giving rise to a random pattern similar to that shown in Fig. 1(e). This is because in an individual nanotube the breakdown occurs at the defect site due to Joule heating and oxidation at a critical temperature of $\sim 600{ }^{\circ} \mathrm{C}$ (Refs. 16 and 22) and one would expect that defects are randomly distributed. In contrast, our experiments demonstrate that the breaking of one nanotube affects the neighboring intact nanotubes in a correlated fashion forcing them to break in the vicinity of its broken region leading to the formation of a continuous channel similar to that shown in Fig. 1(f).

In order to examine what causes such a correlated breaking of SWNT, we performed breaking of arrays with varying SWNT density (Fig. 2) with an average internanotube separations ranging from 500 to $50 \mathrm{~nm}$. At large internanotube separation, the nanotube breaking occurs at random positions without any noticeable correlation between the breaking of neighboring nanotubes [Figs. 2(a) and 2(b)]. Figure 2(c) shows the electrical breakdown of nanotubes when the density is $\sim 8 / \mu \mathrm{m}$ with an average internanotube separation of $120 \mathrm{~nm}$. Unlike Figs. 2(a) and 2(b), a pattern is becoming visible. The pattern becomes much clearer with increasing nanotube density [Figs. 2(d) and 2(e)]. It is clear that as the nanotubes become closer to each other, the breaking of one nanotube affects the neighboring tubes in a correlated fashion. Our results indicate that a noticeable correlation develops when the separation between nanotubes is less than $120 \mathrm{~nm}$ (array density $>8 \mathrm{SWNT} / \mu \mathrm{m}$ ).
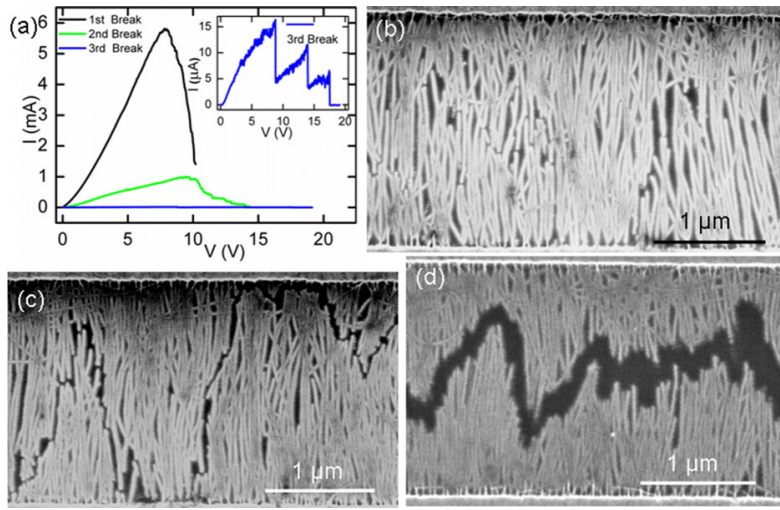

FIG. 3. (Color online) The controlled breaking of an array with $30 \mathrm{SWNT} / \mu \mathrm{m}$. (a) $I-V$ curves show three successive electrical breakdown steps. Inset shows $I-V$ curve during the third step. (b) SEM image after the first breakdown. A small gap has appeared at random locations in the array of SWNT although no clear pattern can be seen. (c) SEM image after second breakdown showing a clear pattern due to correlated breaking of SWNT. (d) SEM image after the final breakdown shows a wider continuous channel.

In order to further investigate the correlated nature of the breaking and how the nanotube breaking progresses, we performed electrical breakdown of SWNT arrays in three successive steps. Figure 3(a) shows the $I$ - $V$ characteristics of a representative array with $\sim 30 \mathrm{SWNT} / \mu \mathrm{m}$. In the first two steps, the current was allowed to drop approximately one order of magnitude from its peak value after which the voltage was swept back to zero. After the third (final) breakdown, current drops to zero [Fig. 3(a) inset]. SEM image taken immediately after each breaking is shown in Fig. 3(b)-3(d). After the first break, quite a few SWNTs are broken at random places all over the network [Fig. 3(b)]. However, no clear pattern is observed. After the second breakdown of the same network, we estimate that more than $90 \%$ of the nanotubes are broken and a clear correlated pattern develops [Fig. 3(c)]. During the third breakdown, the remaining nanotubes get broken and the nanofissure gets wider [Fig. 3(d)]. Since the array contains both metallic and semiconducting nanotubes, our results demonstrate that the semiconducting nanotubes are not immune to correlated breakdown.

We now examine the cause for the correlated breakdown and formation of nanofissure. The correlations could be regarded as an intrinsic feature of sufficiently dense networks or could be attributed to the transfer of heat produced during the burning of a nanotube to the neighboring tubes. ${ }^{5}$ The latter mechanism, however, can be ruled out as it is not supported by the experimental data. Indeed, if this was the case then switching the bias voltage off after the first break (see Fig. 3) would interrupt the formation of the correlated pattern and the nanotube network would proceed breaking at random positions after restoring the high bias voltage. The experiments, however, show that even after restoring the high bias voltage the breaking keeps its continuous correlated form [Fig. 3(d) and 3(e)], which means that heat transfer cannot be responsible for the correlated breakdown. This suggests that the correlation is a manifestation of an intrinsic feature of a sufficiently dense network. We propose that the correlations are caused by the electrostatic field produced around the gap in the broken nanotube. While this field does not create a current, it alters the electron flow in the neighboring intact nanotubes thus affecting the distribution of the 

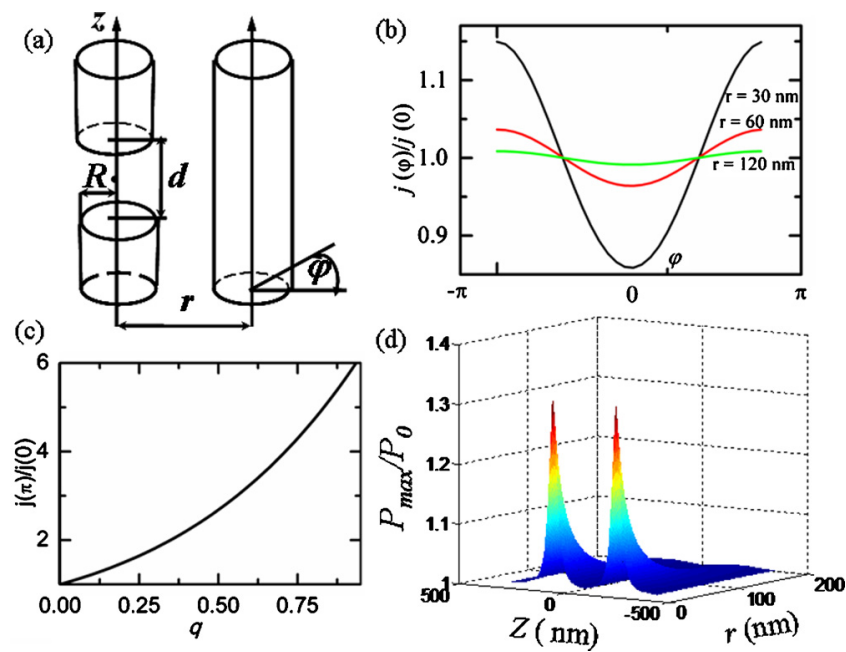

FIG. 4. (Color online) The effect of the break on the intact nanotube. (a) Geometry of the problem. (b) The angular variation in the current density at $z=-d / 2$ (the voltage $V=8 \mathrm{~V}$, the radius of the nanotube $R=1.5 \mathrm{~nm}$, the width of the gap $d=300 \mathrm{~nm}$ ) for different distances between the broken and intact nanotubes $r=30,60$, and $120 \mathrm{~nm}$. (c) Dependence of the ratio of the maximum and minimum values of the current densities on $q$. (d) The ratio of the maximal power of Joule heating due to the current distribution $P_{\max }$ and the power in the case when the broken nanotube is absent $P_{0}$ as a function of the distance between the broken and intact nanotubes and the position along the nanotube.

intensity of Joule heating. The most significant effect can be expected across the gap in the broken nanotube. This promotes the breaking of yet intact nanotubes near already existing breaks thus leading to the formation of a nanofissure.

In order to show the mechanism in more detail, we consider a single nanotube that is broken at the point $z_{0}=0$ [see Fig. 4(a)] and analyze its effect on the current distribution in a neighboring intact nanotube. Under the action of the external bias field the electric charge in the broken nanotube redistributes and creates an induced potential, which breaks the cylindrical symmetry of the intact nanotube. Assuming that the scattering effect of the induced potential is small we find for the current density in the intact nanotube $j(z, \varphi)$ $=\left|\chi_{0}(z, \varphi)\right|^{2} j_{0}$, where $j_{0}$ is the density in the absence of the broken tube and $\chi_{0}(z, \varphi)$ is a solution of an equation of the Mathieu type (see Ref. 21). Remarkably, the specific form of the spatial variation in the current density depends on the single parameter $q(z)=\left(4 m R^{2} / \hbar^{2}\right) U_{2}(-z)$, which is a function of the system parameters, such as the geometrical sizes and the applied voltages. $R$ is the radius of the nanotube, $U_{2}(z)=e Q R r\left(\rho_{+}^{-3}-\rho_{-}^{-3}\right), \rho_{ \pm}=\sqrt{(z \pm d / 2)^{2}+r^{2}}, d$ is the width of the gap, $r$ is the internanotube separation, and $Q$ is the induced charge, which is assumed to concentrate mostly near the edges of the gap. The induced charge is estimated by taking into account that $d \gg R$, which yields $Q=V R$, where $V$ is the applied external voltage.

Figure 4(b) shows the angular dependence of the current density for different $r$. Its variation can be characterized by the ratio of the densities along the opposite sides of the intact nanotube $j(\varphi=\pi) / j(\varphi=0)$ plotted in Fig. 4(c) as a function of $q$. In the shown range this ratio is well approximated by $j(\pi, z) / j(0, z) \approx \exp [2 q(z)]$, which yields the explicit dependence of the current amplitude on the system parameters $j_{\max } \approx j_{0}\{1+\tanh [q(z)]\}$. The spatial variation in the current density leads to an inhomogeneous distribution of the intensity of the Joule heating $P(z, \varphi) \propto j^{2}(z, \varphi)$. In Fig. 4(d), we show the maximal power $P_{\max } \propto j_{\max }^{2}$ as a function of the coordinate along the intact nanotube and its distance from the broken nanotube. It demonstrates that across the gap the Joule heating may noticeably exceed the one in the case of absent broken nanotube thus promoting breaking in this region. At the same time the effect drops fast with the intertube separation, implying that the correlations become significant only in sufficiently dense networks.

In conclusion, we demonstrated nanofissure formation due to a correlated electrical breakdown of sufficiently densed SWNT aligned array. The physical origin of the correlation is in the electrostatic field of the broken nanotubes that produces locally inhomogeneous current and Joule heating distributions in the neighboring intact nanotubes triggering their breakdowns in the vicinity of the broken tubes. Our results suggest that densely aligned SWNT array behaves like a correlated solid. Since the correlated breakdown is of electrostatic origin, such a phenomenon should be visible in other one-dimensional (1D) arrays systems involving nanowires, and graphene nanoribbons. The results presented here will have significant impact in understanding and controlling electrical properties, stability, thermal management, and development of fault-tolerant nanocircuits in arrays of 1D nanostructures.

This work was partially supported by the U.S. NSF under Grant Nos. ECCS 0748091 (CAREER) to S.I.K., ECCS0901784 to M.N.L. and AFOSR under Grant No. FA955009-1-0450 to MNL.

${ }^{1}$ P. G. Collins, M. Hersam, M. Arnold, M. R. Martel, and P. Avouris, Phys. Rev. Lett. 86, 3128 (2001).

${ }^{2}$ P. Avouris, Z. Chen, and V. Perebeinos, Nat. Nanotechnol. 2, 605 (2007). ${ }^{3}$ A. Javey, J. Guo, Q. Wang, M. Lundstrom, and H. Dai, Nature (London) 424, 654 (2003).

${ }^{4}$ C. Rutherglen, D. Jain, and P. Burke, Nat. Nanotechnol. 4, 811 (2009).

${ }^{5}$ C. Rutherglen, D. Jain, and P. Burke, Appl. Phys. Lett. 93, 083119 (2008).

${ }^{6}$ C. Kocabas, H.-s. Kim, T. Banks, J. A. Rogers, A. A. Pesetski, J. E. Baumgardner, S. V. Krishnaswamy, and H. Zhang, Proc. Natl. Acad. Sci. U.S.A. 105, 1405 (2008).

${ }^{7}$ S. J. Kang, C. Kocabas, T. Ozel, M. Shim, N. Pimparkar, M. A. Alam, S. V. Rotkin, and J. A. Rogers, Nat. Nanotechnol. 2, 230 (2007).

${ }^{8}$ F. N. Ishikawa, H.-k. Chang, K. Ryu, P.-c. Chen, A. Badmaev, L. G. De Arco, G. Shen, and C. Zhou, ACS Nano 3, 73 (2009).

${ }^{9}$ K. Ryu, A. Badmaev, C. Wang, A. Lin, N. Patil, L. Gomez, A. Kumar, S. Mitra, H.-S. P. Wong, and C. Zhou, Nano Lett. 9, 189 (2009).

${ }^{10}$ S. Kim, S. Ju, J. H. Back, Y. Xuan, P. D. Ye, M. Shim, D. B. Janes, and S Mohammadi, Adv. Mater. (Weinheim, Ger.) 21, 564 (2009).

${ }^{11}$ P. Stokes, E. Silbar, Y. M. Zayas, and S. I. Khondaker, Appl. Phys. Lett. 94, 113104 (2009).

${ }^{12}$ M. Engel, J. P. Small, M. Steiner, M. Freitag, A. A. Green, M. C. Hersam, and P. Avouris, ACS Nano 2, 2445 (2008).

${ }^{13}$ M. C. LeMieux, M. Roberts, S. Barman, Y. W. Jin, J. M. Kim, and Z. Bao, Science 321, 101 (2008).

${ }^{14}$ Q. Cao and J. A. Rogers, Adv. Mater. (Weinheim, Ger.) 21, 29 (2009)

${ }^{15}$ P. G. Collins, M. S. Arnold, and P. Avouris, Science 292, 706 (2001).

${ }^{16}$ E. Pop, Nanotechnology 19, 295202 (2008).

${ }^{17}$ H. Maune, H.-Y. Chiu, and M. Bockrath, Appl. Phys. Lett. 89, 013109 (2006).

${ }^{18}$ S. W. Hong, T. Banks, and J. A. Rogers, Adv. Mater. (Weinheim, Ger.) 22 1826 (2010)

${ }^{19}$ C. Wang, K. Ryu, L. G. De Arco, A. Badmaev, J. Zhang, X. Lin, Y. Che, and C. Zhou, Nano Res. 3, 831 (2010).

${ }^{20}$ S. Shekhar, P. Stokes, and S. I. Khondaker, ACS Nano 5, 1739 (2011).

${ }^{21}$ See supplementary material at http://dx.doi.org/10.1063/1.3600664 for assembly of SWNTs with controlled density, and detailed theoretical formalism.

${ }^{22}$ K. Hata, D. N. Futaba, K. Mizuno, T. Namai, M. Yumura, and S. Iijima, Science 306, 1362 (2004). 